\title{
ANALISIS DE SENSIBILIDAD PARA EL MDVRPPC MULTI-OBJETIVO QUE CONSDERA COSTO E IMPACTO AMBIENTAL.
}

\section{SENSIBILITY ANALYSIS FOR THE MULTI-OBJECTIVE MDVRPPC THAT CONSIDERING COST AND ENVIRONMENTAL IMPACT.}

\author{
Ing. Alejando Ospina Castaño*, PhD. Eliana Mirledy Toro-Ocampo** \\ PhD. Ramón Alfonso Gallego-Rendón ${ }^{* * * *}$ \\ *,*** Universidad Tecnológica de Pereira, Facultad de Ingenierías, Programa de \\ Ingeniería Eléctrica. \\ Cra. 27 \#\#10-02, Pereira, Risaralda, Colombia \\ PBX: +57 63137300 \\ E-mail: \{aopsina, ragr\}@utp.edu.co. \\ ** Universidad Tecnológica de Pereira' Facultad de Ciencias Empresariales , Programa \\ de Ingeniería Industrial Cra. 27 \#\#10-02, Pereira, Risaralda, Colombia \\ PBX: +57631373205 \\ E-mail: elianam@utp.edu.co.
}

\begin{abstract}
Resumen: En este artículo se presenta un modelo matemático para resolver el problema de ruteo verde usando flota propia y flota subcontratada considerando múltiples depósitos (GMDVRPPC). El modelo planteado es del tipo bi-objetivo, donde se consideran tanto costos operativos como impacto ambiental, medido a través del consumo de combustible de los vehículos. El estudio realiza un análisis de sensibilidad que considera la topografía del terreno (grados de inclinación) y estado de la vía, para lo cual se calcula una distancia e inclinación virtual de los arcos. Para validar la propuesta se usan instancias de la literatura especializada, a fin de establecer el mejor modo de operación que relaciona el número de vehículos y tiempo de entrega de acuerdo con la inclinación y estado de la vía.
\end{abstract}

Palabras clave: condiciones de la vía, flota propia, flota subcontratada, múltiples depósitos, ruteo verde,

\begin{abstract}
In this article a mathematical model is presented to solve the Green- Multidepot vehicle routing problem with private fleet and common carrier (GMDVRPPC). The model proposed is the bi-objective type, where both operating costs and environmental impacts are considered, measured through the consumption of fuel from the vehicles. The study performs a sensitivity analysis that considers the topography of the Path (degrees of inclination) and the state of the road, for which a distance and virtual inclination of the arcs are calculated. In order to validate the proposal, instances of specialized literature are used, in order to establish the best mode of operation that relates the number of vehicles and delivery time according to the inclination and state of the way.
\end{abstract}

Keywords: Common Carrier, Green routing, private fleet, path conditions.

\section{INTRODUCCIÓN}

Uno de los aspectos más importantes en la planeación de la cadena de suministro tiene relación con la distribución del producto final. Muchas compañías realizan esta actividad a través de una flota de vehículos propios y/o de vehículos subcontratados. En el problema de ruteo de vehículos considerando flota propia y flota subcontratada, vehicle routing problem with private fleet and 
common Carrier (VRPPC) es necesario determinar cómo se confeccionan las rutas y como se asignan a los diferentes tipos de flota minimizando los costos de distribución (Euchi, 2017).

La experiencia ha mostrado que el alquiler de una flota externa para pequeñas cantidades de mercancía en áreas locales, tal como se requiere en la logística de última milla, usualmente reduce el número de vehículos necesarios para atender la demanda de los clientes, lo que impacta en la reducción de los costos de distribución de las empresas (Dua et al., 2014).

La temática de ruteo de vehículos ha recibido amplia atención de los investigadores por su gran cantidad de aplicaciones, así como por la naturaleza de su complejidad computacional, ya que representa un desafío en el campo de la optimización matemática tanto exacta como aproximada. Corresponde a una gran variedad de problemas donde se consideran diferentes topologías en la red de distribución relacionadas con el nivel de operación de las empresas, donde es posible considerar uno o varios depósitos que deben atender un conjunto de clientes con determinada demanda (Toro et al., 2016).

El problema de ruteo de vehículos con un depósito y una flota de vehículos con capacidad limitada donde se conoce la demanda de los clientes se denomina capacitated vehicle routing problem (CVRP) (Ocampo, 2016) y cuyo objetivo es minimizar los costos de las rutas. Cuando la complejidad de la operación aumenta aparece el problema de ruteo de vehículos considerando múltiples depósitos, Multidepot vehicle routing problema (MDVRP) el cual ha sido resuelto de forma eficiente tanto a través de metodologías exactas, como de metodologías aproximadas (Rendón et al. 2015). Cuando la atención a los clientes se efectúa usando flota subcontratada de forma exclusiva se denomina Multi Depot Open Vehicle (MDOVRP) el cual corresponde a una extensión del problema de ruteo abierto OVRP y una variante del MDVRP (Ospina-Toro et al., 2018), que ha sido resuelto eficientemente mediante técnicas metaheurísticas tales como algoritmo genético (Liu et al., 2014) Búsqueda Tabú y mediante estrategias exactas como la planteada por Lalla-Ruiz et al., 2016.

Cuando se considera el uso combinado de vehículos propios y subcontratados se plantea el VRPPC de gran interés porque aparece en las organizaciones cuando la demanda excede la disponibilidad de la flota propia y es necesario subcontratar un conjunto de vehículos que atienden la demanda adicional. Si se consideran múltiples depósitos se configura el
Multi-depot vehicle routing problem with private fleet and common Carrier (MDVRPPC) (ToroOcampo et al., 2016).

En los últimos años las organizaciones han manifestado su interés por generar soluciones que sean satisfactorias desde el punto de vista económico, pero sin omitir la sostenibilidad ambiental. En la temática de ruteo se ha planteado variantes verdes donde se considera la minimización de las emisiones contaminantes medidas en la cantidad de gasolina consumida en las rutas. Pueden ser encontradas en la literatura especializada como Pollutant Routing Problem (PRP) o como Green vehicle routing problem (GVRP) (Bektaş, T., \& Laporte,2011; Toro et. al, 2015).

En esta propuesta se toma como base el modelo matemático planteado en Toro et al., 2017 donde se presenta un modelo matemático flexible que representa diferentes aproximaciones de los VRP, de tal forma que a través de modificaciones en las variables y restricciones se obtienen variantes como el MDVRPPC, caso de este estudio. La función de impacto se modela a través de un estudio de fuerzas en equilibrio y en función de la carga transportada por el vehículo y la distancia viajada.

Las contribuciones de este artículo son: Primero presentar el problema de ruteo verde, GreenMultidepot Vehicle Routing problem With private fleet and common carrier (GMDVRPPC) como alternativa en la gestión logística. Segundo, presentar y desarrollar una metodología bi-objetivo que considera las condiciones de pendiente y estado de la vía y sus efectos sobre la minimización de la distancia recorrida, así como el consumo de combustible medido en $\mathrm{Kg}$ de $\mathrm{CO} 2$.

La organización del documento es la siguiente: En la Sección 2, se describe el GMDVRPPC y se presenta el modelo matemático. En la sección 3 se presenta la función objetivo ambiental y las consideraciones de la pendiente y condiciones de la vía. Finalmente se presentan los resultados y conclusiones de la estrategia propuesta.

\section{PROBLEMA DE RUTEO VERDE GMDVRPPC}

El GMDVRPPC se define mediante un grafo doble, uno asociado a los arcos que definen las rutas recorridas con la flota propia y el otro asociado a los arcos recorridos usando los vehículos subcontratados. Se consideran los siguientes aspectos: $i$ ) Los vehículos propios deben 
regresar a el depósito donde inició su ruta. ii) Los vehículos subcontratados, finalizan la ruta en el último cliente atendido. iii) No se debe exceder la capacidad de los vehículos y depósitos. iv) Se debe atender completamente la demanda de cada nodo.

\subsection{Modelo radial del MDVRPPC.}

El modelamiento matemático del MDVRPPC se inspira en las redes de distribución de energía eléctrica (Lavorato, et al., 2012). A partir del cual Toro-Ocampo, 2016 realiza una analogía a las redes de transporte. Se formula un problema lineal entero mixto, el cual es definido por las ecuaciones (1) -(28). El modelo considera conjunto de depósitos $I$, un conjunto de clientes $J$ y en general un conjunto de nodos $V=I U J$.

\section{Variables}

$x_{i j} \quad$ Se activa cuando el arco $i ; j \in V$ es recorrido por un vehículo propio.

$s_{i j} \quad$ Se activa cuando el arco $i ; j \in V$ es recorrido por un subcontratado.

$y_{i} \quad$ Variable binaria que indica la apertura del centro de distribución. $i \epsilon I$

$f_{i j} \quad$ Define si el consumidor $j$ es atendido por una ruta del depósito $i \epsilon I$.

$z_{j} \quad$ Determina si el consumidor $j \in J$ es el último de la ruta en ser atendido.

$a_{i j} \quad$ Variable binaria de conexión al depósito donde inicia la ruta.

$t_{i j} \quad$ Indica la cantidad de carga entre los nodos $i, j$ usando flota propia

$l_{i j} \quad$ Indica la cantidad de carga en el arco $i$ y $j$ transportada por el vehículo subcontratado.

\section{Parámetros}

$W_{i} \quad$ Capacidad del depósito

$Q \quad$ Máxima carga que puede ser transportada por un vehículo.

$D_{j} \quad$ Demanda de cada cliente

$C_{i j} \quad$ Costo de viajar entre los nodos $i$ y $j$

$P \quad$ Factor de penalización para arcos transitados por flota

$\mathrm{NVa} \quad$ Número de vehículos disponibles

$$
\min \Psi_{1}=\sum_{i j \in V} c_{i j} x_{i j}+\sum_{\substack{i \in I \\ j \in J}} c_{i \mathfrak{i j}} a_{i \mathfrak{i f}}+P \sum_{\substack{i \in V \\ j \in V}} c_{i j} s_{i j}
$$

Sujeto a:

$$
\begin{aligned}
& \sum_{i \in V} x_{i f}+\sum_{i \in V} s_{i f}=1 \\
& \forall j \in J \\
& \sum_{k \in !} x_{j k}+\sum_{i \in I} a_{i j}=\sum_{i \in V} x_{i j} v \quad \forall j \in J \\
& \sum_{j \in]} x_{i f}=\sum_{j \in j} a_{i j}, \quad \forall i \in I \\
& \sum_{k \in]} s_{j k} \leq \sum_{i \in V} s_{i j} v \quad \forall j \in J \\
& x_{i j}+x_{j i}+s_{i j}+s_{j i} \leq 1, \quad \forall i, j \in V \\
& \sum_{\substack{i \in j \\
i \neq j}} t_{i j}+l_{i f}=\sum_{\substack{k \in V \\
k \neq j}}\left(t_{j k}+l_{j k}\right)+D_{j}, \quad \forall j \in J \\
& \sum_{i \in j} x_{i j}+s_{i f}=\operatorname{card}(j), \quad \forall j \in J \\
& \sum_{i \in I} f_{i j} \leq 1, \quad \forall j \in J \\
& t_{i j} \leq Q x_{i j}, \quad \forall i, j \in V \\
& I_{i f} \leq Q s_{i j}, \quad \forall i, j \in V \\
& \sum_{i \in j} t_{i f}+l_{i f} \leq w_{i} y_{i}, \quad \forall i \in I \\
& \sum_{i \in V} s_{i j}+\sum_{k \in V} x_{j k}=1-z_{j}, \quad \forall j \in J \\
& 1+a_{i j} \geq f_{i f}+z_{j} \\
& -\left(1-x_{j u}-x_{u j}\right) \leq f_{i f}-f_{i w} \\
& f_{\text {if }}-f_{\text {iu }} \leq\left(1-x_{f u}-x_{u j}\right), \quad \forall i \in I, \forall j, u \in V \\
& f_{i j} \geq x_{i j}, \quad \forall i \in I, \forall j \in J \\
& \sum_{i \in I} y_{i} \geq \sum_{j \in J} D_{j} / \sum_{i \in I} w_{i}, \quad \forall i \in I \\
& \sum_{i \in J} x_{i j}+s_{i j} \leq \sum_{f \in J} D_{j} / Q v \quad \forall i \in I \\
& \sum_{\substack{j \in j_{i j} \\
i \in V}} a_{i j} \leq N V_{a} \\
& x_{i j} \in\{0,1\}, \quad \forall i, j \in V \\
& s_{i j} \in\{0,1\}, \quad \forall i, j \in V
\end{aligned}
$$

$(\mathbb{i} 4 \mathcal{F} \boldsymbol{I}, \forall j \in J$

$\forall i(i))_{v} \forall j, u \in V$ 


$$
\begin{array}{cr}
y_{i} \in\{0,1\}, & \forall i \in V \\
f_{i j} \in\{0,1\}_{v} & \forall i \in I_{v} \forall j \in V \\
z_{j} \in\{0,1\}_{v} & \forall j \in J \\
a_{i j} \in\{0,1\}_{v} & \forall i \in I_{v} \forall j \in V \\
t_{i j} \in R_{v} & \forall i, j \in V \\
l_{i j} \in R_{s} & \forall i_{v} j \in V
\end{array}
$$

La ecuación (1) minimiza los costos operativos asociados al recorrido de cada arco por flota propia y subcontratada. (2) explica que cada cliente es visitado una sola vez por flota propia o subcontratada. Garantiza que todos los nodos visitados por la flota propia son de grado 2 (3). La restricción (4) explica que el número de vehículos propios que salen de cada depósito sea igual al número de vehículos propios que llegan a cada depósito. Si un cliente es visitado por flota subcontratada, entonces se debe salir del mismo con flota subcontratada siempre y cuando este no sea un nodo final según (5). En (6) se obliga a que cada vehículo viaje en una sola dirección sin considerar la asimetría de la ruta. La restricción (7) o de balance nodal, afirma que la sumatoria de carga que entra a un nodo debe ser igual a la suma de la carga que sale del nodo y la que se queda allí. (8) Impone la característica de radialidad que posee la red de transporte. (9) Garantiza que un nodo de demanda debe estar conectado a un centro de distribución. Cada flota usada en la operación sea propia o subcontratada, no debe exceder su capacidad máxima en el transporte de carga de acuerdo con (10) y (11). Con (12) se garantiza que la capacidad del depósito no exceda su límite de carga. En (13) se identifica el nodo final sin arco de salida para flota propia, siempre y cuando no se ingrese con flota subcontratada. Si j es un cliente terminal visitado por flota propia, entonces (14) obliga a que exista un arco de retorno. Con (15) y (16) se identifica en la red de transporte el depósito de inicio para vehículos propios, tal que se pueda activar el arco de retorno. Si el arco entre i y j está activo, entonces se garantiza por medio de (17) que la demanda de un cliente $j$ está conectada con un centro de distribución $i$. Con (18) se establece la cantidad mínima de depósitos que deben ser instalados con base a la relación entre demanda total y capacidad del depósito. (19) Determina la cantidad mínima de vehículos usados en la operación a partir de la relación entre demanda total y la capacidad del vehículo. En (20) se define el número máximo de flotas propias que van a ser usadas en la operación. De (21) a (28) se define la naturaleza las variables. El modelo es resuelto y usando la técnica Epsilon Constraint, (Ehrgott y Gandibleux, 2003).

\section{MODELO DE IMPACTO AMBIENTAL}

El modelo de impacto ambiental es expuesto en (Toro-ocampo, 2016). Este modelo se basa en un sistema de equilibrio como se representa en la figura 1, donde $F_{M}$ es la fuerza que ejerce el motor en la rueda, para darle movimiento al vehículo. $\beta_{i j}$ representa el ángulo de inclinación promedio que posee la vía en el tramo $i, j$. El peso del vehículo está representado por $m g$. $N$ es la fuerza normal de la superficie. $v$ es la velocidad promedio del vehículo entre el tramo $i j$ y $d_{i j}$ es la distancia promedio que hay entre el punto $i$ y el punto $j$.

La ecuación (29) representa las fuerzas que se oponen al movimiento del vehículo.

$$
\vec{F}_{R}=\vec{F}_{R_{d} \text { llantas }}+\vec{F}_{R_{d} \text { viento }}+\vec{F}_{R_{d} \text { intemas }}+\frac{m v_{i j}^{2}}{2 d_{i j}}
$$

Como se muestra en la figura 1 efectuando un balance de fuerzas en los ejes $x$ y $y$ se obtiene la fuerza que el motor ejerce en las ruedas, la cual es dada en función de la masa total del vehículo, la gravedad, la pendiente, constante que depende del terreno, la fuerza del viento, las fuerzas internas y la fuerza para lograr la velocidad de estado estacionario expresada en (30).

$$
\begin{gathered}
F_{M}=\left(m g \cos \beta_{i j}\right) b+F_{\text {Rviento }}+F_{R_{j} \text { internas }} \\
+\frac{m v_{i j}^{2}}{2 d_{i j}}+m g \sin \beta_{i j}
\end{gathered}
$$

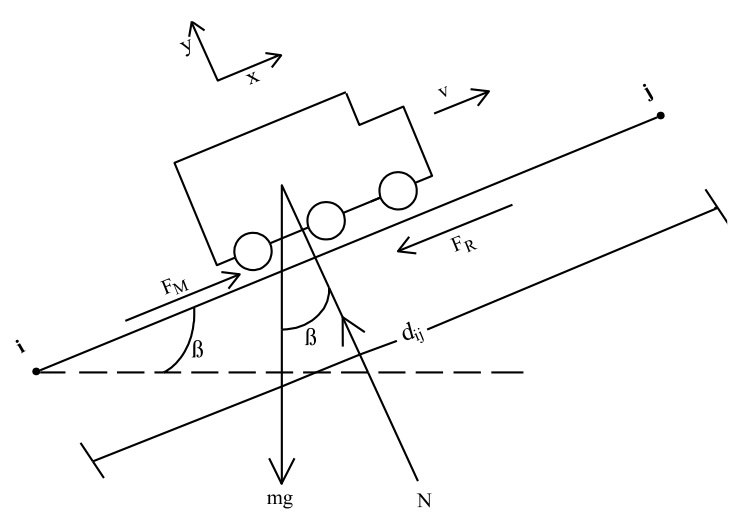

Figura 1. Sistema de equilibrio estático para un vehículo en pendiente. Toro-Ocampo,2016 
La fuerza convertida en trabajo así: $U_{i j=} F_{M} d_{i j}$. La masa está compuesta de dos partes, la primera la que hace relación al vehículo (constante) y la segunda a la carga transportada en el tramo $i-j$ (variable) como se expresa en (31).

$$
U_{i f}=\alpha_{i j} d_{i f}+\gamma_{i j} t_{i j} d_{i f}
$$

La constante $\alpha_{i j}$ (33) tiene unidades de Joule/Km y está en función de la inclinación promedio entre $i, j$ y el tipo de terreno, las fuerzas de fricción internas además de la fuerza requerida por el mismo para alcanzar la velocidad de estado estacionario.

$$
\begin{aligned}
\alpha_{i j}=\alpha= & m_{0} g\left(b \cos \beta_{i j}+\sin \beta_{i j} \frac{v_{i j j}^{2}}{2 g d_{i j}}\right)+ \\
& +F_{R, \text { viento }}+F_{R, \text { internas }} \\
\gamma_{i j}=\gamma & =g\left(b \cos \beta_{i j}+\sin \beta_{i j} \frac{v_{i j}^{2}}{2 g d_{i j}}\right)
\end{aligned}
$$

La constante $\alpha_{i j}$ tiene unidades de Joule/Km y está en función de la inclinación promedio entre $i, j$ y el tipo de terreno, las fuerzas de fricción internas y la fuerza requerida por el mismo para alcanzar la velocidad de estado estacionario. La constante $\gamma_{\mathrm{ij}}$ tiene unidades de Joule/ $\mathrm{kg} * \mathrm{~km}$ y está en función del ángulo promedio de inclinación entre $i, j$ y el tipo de terreno y se representa en (34).

Para determinar los arcos activos, se asignan las variables binarias $a_{i j}, x_{i j}, s_{i j}$ (35) El trabajo total requerido por un vehículo para completar una ruta se da por la sumatoria de los trabajos realizados en cada tramo de ruta propia (36) y en la subcontratada (37).

$$
\begin{aligned}
& U_{i j}\left(t_{i j p} l_{i j}, x_{i j} s_{i j} a_{i j}\right)=\alpha d_{i j} x_{i j}+\alpha d_{i j} a_{i j}+ \\
& +\gamma t_{i j} d_{i j} x_{i j}+\alpha d_{i j} s_{i j}+\gamma l_{i j} d_{i j} s_{i j} \\
& \sum_{i_{j} j \in V} U_{i j-1}=\alpha\left(\sum_{i_{j j} \in V} d_{i j} x_{i j}+\sum_{i_{i j} \in V} d_{i j} a_{i j}\right)+ \\
& +\gamma\left(\sum_{i_{0 j} \in V} d_{i j} t_{i j}\right) \\
& \sum_{i_{l} j \in V} U_{i j-2}=\alpha\left(\sum_{i_{l} j \in V} d_{i j} S_{i j}\right) \\
& +\gamma\left(\sum_{i_{i j} \in V} d_{i j} l_{i j}\right)
\end{aligned}
$$

La función objetivo de impacto ambiental se calcula mediante (38) usando factores de conversión $E_{l}$ en (galones/J) y (kgCO2/galones) para (36)y (37). Donde $E=E_{1} * E_{2}$. La ecuación (38) representa la función objetivo de impacto ambiental.

$$
\min \Psi_{2}=E \times\left(\sum_{i_{0} j \in V} U_{i j-1}+\sum_{i_{0}, j \in V} U_{i j-2}\right)
$$

\subsection{Modelo de distancia equivalente por efecto pendiente.}

Para considerar una vía con diferentes grados de inclinación y estado del arco, los coeficientes $\alpha$ y $\gamma$ de la ecuación de trabajo son variados en función del ángulo promedio $\beta_{i j}$ y el coeficiente de fricción $b$, la figura 2 representa este concepto.

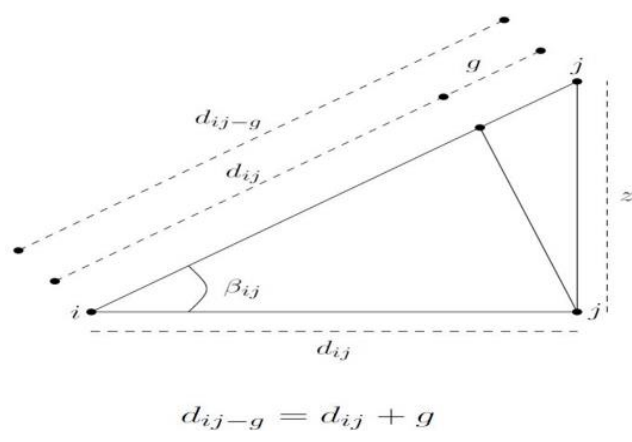

Figura 2. Corrección de distancia-pendiente.

Al ser variado el ángulo del arco, se varia la distancia $d_{i j}$, además se considera $g$ como factor de corrección por efecto pendiente (39) (Carciente, 1980)

$$
d_{i j-g}=\left(\frac{100+g}{100}\right) d_{i j}
$$

La expresión (40) puede ser expresada en función del ángulo $\beta_{i j}$ y la distancia a cero grados $d_{i j}$,

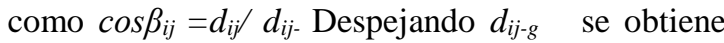
(40)

$$
\left(\frac{1}{\cos \beta_{i j}}\right) \simeq\left(\frac{100+g}{100}\right)
$$

\subsection{Método de bruce, o método de la distancia virtual.}

Se plantea mediante (41) la distancia virtual con base en la figura 3 (Carciente, 1980; Gárdenas Grisales, 2013)

$$
d_{0-i j}=d_{i j}+k \times \sum z
$$


Se define el inverso del coeficiente de tracción $k$ como: $k=1 / \varphi$, la cual corresponde a una constante adimensional que depende del estado de la vía o del tipo de capa de rodadura utilizada para el terreno. La sumatoria de las $z$, corresponde a la suma de las longitudes verticales en el eje de elevación $z$ de los tramos en contrapendiente, donde el número de contrapendientes entre $i-j$ y $j-i$ no es el mismo, $d_{0-i j} \neq d_{0-j i}$.

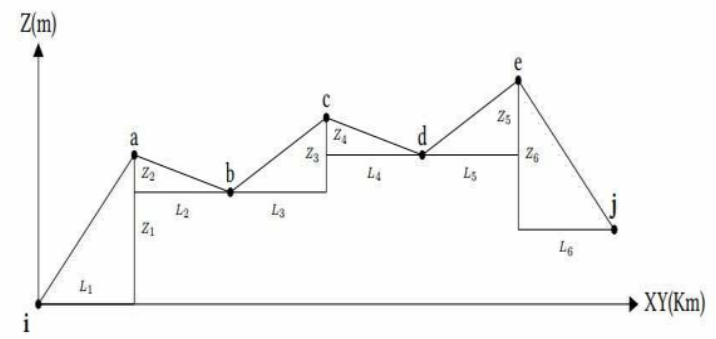

Figura 3. Relieve del arco $i$-j.

El trabajo de flota propia $U_{i j-1}$ y $U_{i j-2}$ de flota la subcontratada se obtiene en (42), lo que representa el trabajo virtual para que un vehículo se desplace. Uo-ij es el trabajo virtual requerido por el vehículo.

$$
\begin{gathered}
U_{0-i j}=\left(d_{i j}+k \sum z\right) \varphi \\
{\left[\alpha x_{i j}+\alpha a_{i j}+\alpha s_{i j}+\gamma t_{i j} x_{i j}+\gamma l_{i j} s_{i j}\right]}
\end{gathered}
$$

\section{ANALISIS DE RESULTADOS}

Se analizan instancias modificadas de 20 y 30 obtenidas a partir de la instancia principal p01 (Dorronsoro Díaz, n.d.), se usó un computador con sistema operativo Windows 7 de 64 bits con procesador Intel(R) Core(TM) i5-4460S CPU @ $2.90 \mathrm{GHz}$ y una memoria RAM instalada de 12.0 GB.

Se considera Un vehículo de 20 toneladas en promedio consume de $25.3 \mathrm{mpg}$ (millas por galón) equivalente a consumir un galón de gasolina corriente por cada $15.7207 \mathrm{Km}$ recorridos. (UMTRI - University of Michigan Transportation Research Institute, 2014). Según Natural Resources Canada, 2019se considera que un vehículo produce $2.3 \mathrm{Kg} \mathrm{CO}$ por litro de combustible con un costo promedio 8.706449 $\mathrm{Kg} / \mathrm{CO}_{2}$ - galón. El costo de las emisiones corresponde a 0.009 USD por $\mathrm{Kg}-\mathrm{CO} 2$. El precio de un galón de gasolina es de 3.92 USD (GlobalPetrolPrices.com, 2018).

Se asumen todos los arcos con la misma inclinación. La distancia es penalizada usando alguno de los siguientes factores 1/ Cos $\beta_{i j}$ ó $100+\mathrm{g} / 100$. En estas circunstancias el arco es simulado usando una distancia y un ángulo que resulta ser constante. En el caso de distancia virtual tratado en el artículo, se describe el cálculo de distancia y ángulo equivalente, con base en la topografía del terreno, que, a pesar de calcular una distancia plana, representa el equivalente de esfuerzo de tracción dado en energía al recorrer vías con diferentes grados de inclinación y estado de esta. Los rectángulos representan los depósitos de la red, y los círculos los clientes. Como se puede observar en las figuras 4, 5, 6 y 7 el número de vehículos empleados en la operación es mayor en la medida en que la inclinación de la vía es incrementada.

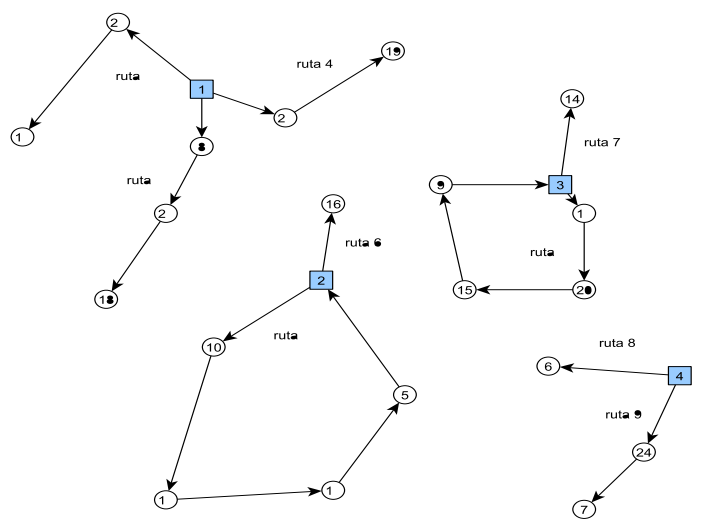

Figura 4. Arriba ruteo a cero grados

Según los resultados en la medida que se incremente la pendiente aumenta el número de vehículos necesarios para atender la demanda. Se consideraron inclinaciones de 0,15 y 20 grados. El aumento en el número de vehículos cuando se incrementa la pendiente se explica como la reducción del esfuerzo de tracción en las pendientes que minimiza el impacto ambiental.

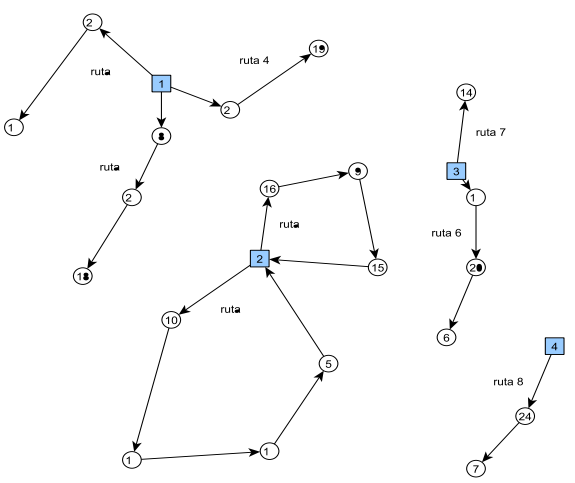

Figura 5. Caso 20C_4D ruteo a 20 grados 
Tabla 2. Resultado para 20 clientes 4 depósitos.

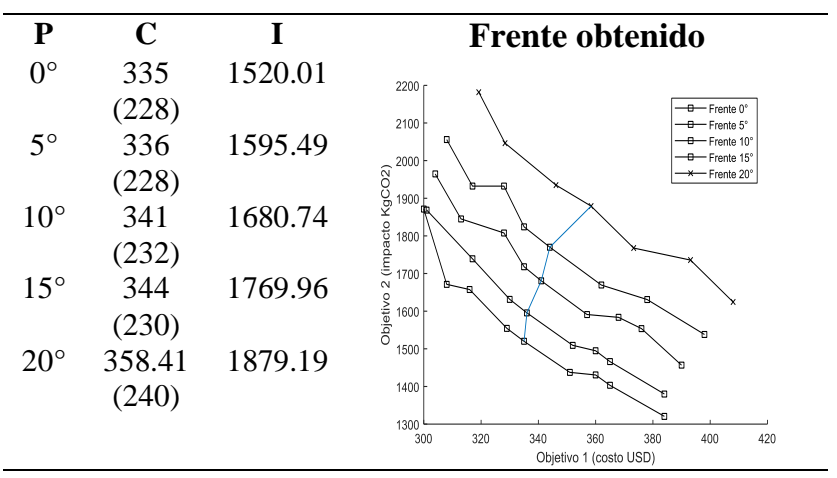
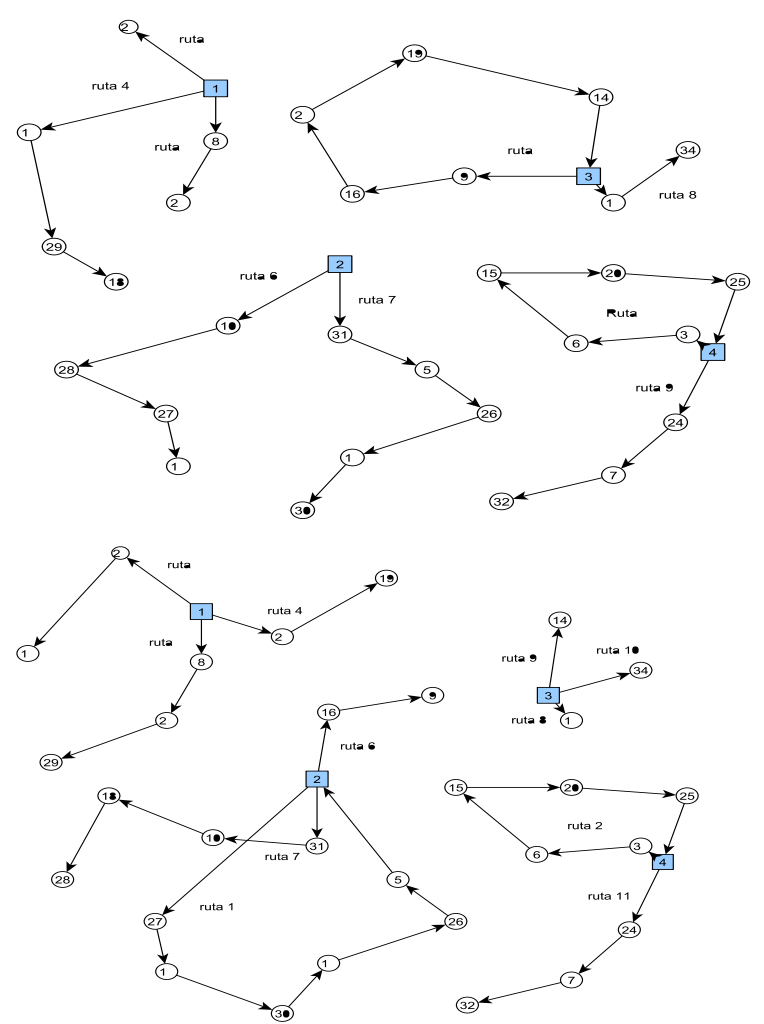

Figura 6. Arriba Caso 30C_4D ruteo a 0 grados, Abajo Caso 30C_4D ruteo a 20 grados

\section{Tabla 3. Resultado para 30 clientes con 4 depósitos}

\begin{tabular}{|c|c|c|c|}
\hline $\mathbf{P}$ & C & I & Frente obtenido \\
\hline $0^{\circ}$ & $\begin{array}{c}474 \\
(358)\end{array}$ & 2028.99 & 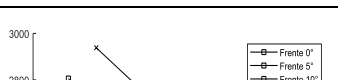 \\
\hline $5^{\circ}$ & $\begin{array}{c}478 \\
(362)\end{array}$ & 2134.53 & 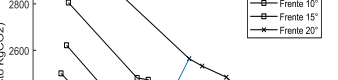 \\
\hline $\begin{array}{c}10 \\
\circ\end{array}$ & $\begin{array}{c}485 \\
(368) \\
\end{array}$ & 2248.48 & \\
\hline $\begin{array}{c}15 \\
\circ\end{array}$ & $\begin{array}{c}491 \\
(372)\end{array}$ & 2357.4 & \\
\hline $\begin{array}{c}20 \\
\circ\end{array}$ & $\begin{array}{c}504 \\
(364)\end{array}$ & 2564 & 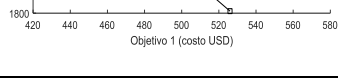 \\
\hline
\end{tabular}

Los casos anteriores son catalogados de baja complejidad matemática y solucionados mediante una técnica exacta. Para casos de mayor complejidad matemática, se recomienda el uso de heurísticas y/o metaheurísticas, que a pesar de no garantizar el óptimo global, encuentran soluciones de buena calidad, en tiempos computacionalmente aceptables. (Alvarez , et. al, 2010; Araque et al., 2017)

\section{CONCLUSIONES}

Se plantea un modelo matemático lineal enteromixto para el GMDVRPPC con un enfoque biobjetivo, minimizando costos de operación y emisiones contaminantes. Se realiza un análisis de sensibilidad con diferentes grados de inclinación y estados de la vía.

Se desarrolla un modelo para el cálculo de la distancia virtual, teniendo en cuenta la inclinación y el estado de la vía. El desarrollo presenta dos alternativas. La primera consiste en modelar el arco con pendiente constante y la segunda con base en el relieve de la vía. A pesar de que la segunda alternativa nos acerca a la realidad, su cálculo es intratable computacionalmente, por tanto, se usa la primera considerando pendientes y estado de la vía similares en todos los arcos.

Con base en los resultados se determinó que el número de vehículos usados en la operación, el costo operativo y las emisiones de $\mathrm{CO} 2$ tienden a aumentar a medida que la pendiente crece. El aumento en el número de vehículos es apreciable para instancias grandes y para cambios bruscos en la pendiente (mayores al 7\%). Pequeños incrementos en la pendiente de la vía no afectan significativamente el ruteo. Sin embargo, el costo y el impacto ambiental si se afectan.

\section{REFERENCIAS}

Alvaréz, D., Toro-Ocampo, E., Gallego- Rendón, R. (2010). Algoritmo GRASP para resolver el problema de asignación de horarios en empresas de demanda variable. Revista Colombiana de Tecnologías de Avanzada (RCTA), ISSN: 1692-7257, 2(16).

Araque, J. A., Rodríguez, J. L. D., \& Guerrero, A. S. (2017). Optimización por recocido simulado de un convertidor multinivel monofásico con modulación pwm sinusoidal de múltiple portadora. Revista Colombiana de Tecnologias de Avanzada, ISSN: 1692- 7257, 1(27) 
Bektas, T., \& Laporte, G. (2011). The pollutionrouting problem. Transportation Research Part B: Methodological, 45(8), 1232-1250.

Carciente, J. (1980). Carreteras estudio y proyecto. (E. V. S.r.1, Ed.) (2nd ed.). Vega, 1980.

Cárdenas Grisales, J. (2013). Diseño geométrico de carreteras (segunda ed). Bogotá: Ecoe Ed.

Dorronsoro Díaz, B. (n.d.). The VRP Web. Retrieved March 7, 2019, from http://www.bernabe.dorronsoro.es/vrp/

Dua, A., Kumar, N., \& Bawa, S. (2014). A systematic review on routing protocols for vehicular ad hoc networks. Vehicular Communications, 1(1), 33-52.

Ehrgott, M., \& Gandibleux, X. (2003). Multiobjective combinatorial optimizationtheory, methodology, and applications. In Multiple criteria optimization: State of the art annotated bibliographic surveys (pp. 369444). Springer, Boston, MA.

Euchi, J. (2017). The vehicle routing problem with private fleet and multiple common carriers: Solution with hybrid metaheuristic algorithm Vehicular Communications, 9, 97-108.

Lalla-Ruiz, E., Expósito-Izquierdo, C., Taheripour, S., \& Voß, S. (2016). An improved formulation for the multi-depot open vehicle routing problem. OR Spectrum, 38(1), 175187.

Lavorato, M., Franco, J. F., Rider, M. J., \& Romero, R. (2012). Imposing radiality constraints in distribution system optimization problems. IEEE Transactions on Power Systems, 27(1), 172-180.

Lopez, L., Hincapié, R., \& R., G. (2011). Planeamiento multiobjetivo de sistemas de distribución usando algoritmos evolutivo NSGA-II. Revista EIA, 8(15), 141-151.

Liu, R., Jiang, Z., \& Geng, N. (2014). A hybrid genetic algorithm for the multi-depot open vehicle routing problem. OR Spectrum, 36(2), 401-421.

Ospina-Toro, D, Toro-Ocampo, E. M., \& GallegoRendón, R. A. (2018). Solución Del Mdvrp Usando El Algoritmo De Búsqueda Local Iterada. Revista Colombiana de Tecnologias de Avanzada, 1(31), 120-127.

Ospina-Castaño, A. (2018). Análisis de sensibilidad para el mdvrppc multiobjetivo que considera costo e impacto ambiental, con diferentes grados de inclinación y estado del arco. Universidad Tecnológica de Pereira. Proyecto de grado

Rendón, R. A. G., Ocampo, E. M. T., \& Zuluaga, A. H. E. (2015). Técnicas Heurísticas y Metaheurísticas. Universidad Tecnológica de Pereira. Vicerrectoría de Investigaciones, Innovación y Extensión. Ingenierías Eléctrica, Electrónica, Física y Ciencias de la Computación.

Toro O., E. M., Escobar Z., A. H., \& Granada E., M. (2015). Literature review on the vehicle routing problem in the green transportation context. Luna Azul, (42), 362-387.

Toro-ocampo, E. M. (2016). Solución del problema de localización y ruteo usando un modelo matemático flexible y considerando efectos ambientales. Universidad Tecnológica de Pereira. Tesis doctoral

Toro-Ocampo, E. M., Franco-Baquero, J. F., \& Gallego-Rendón, R. A. (2016). Modelo matemático para resolver el problema de localización y ruteo con restricciones de capacidad considerando flota propia y subcontratada. Ingeniería, Investigación y Tecnología, 17(3), 357-369

Toro, E. M., Franco, J. F., Echeverri, M. G., \& Guimarães, F. G. (2017). A multi-objective model for the green capacitated locationrouting problem considering environmental impact. Computers \& Industrial Engineering ,110, 114-125.

\section{SITIOS WEB}

GlobalPetrolPrices.com. (2018). Los precios de la gasolina y el diesel por país. Retrieved March 7, 2019, https://es.globalpetrolprices.com/

Natural Resources Canada. (2019). Fuel Consumption Guide.Retrieved March 7, https://www.nrcan.gc.ca/energy/efficiency/ transportation/21002

UMTRI - University of Michigan Transportation Research Institute. (2014). Large drop in fuel economy in September. Retrieved March 7, 2019,http://www.umtri.umich.edu/what-weredoing/news/large-drop-fuel-economy-sept 